\title{
Determinação da doçura ideal em néctar de mamão adicionado de açúcar
}

\author{
Determination of ideal sweetness in nectar of papaya with sugar
}

\author{
Héberly Fernandes Braga ${ }^{\mathrm{I}}$ Ana Carolina Conti-Silva ${ }^{\mathrm{II}}$
}

\section{RESUMO}

O mamão é um fruto cuja polpa possui características sensoriais, químicas e digestivas atrativas, apresentando alta produção no país. Apesar disso, o néctar desse fruto é pouco difundido no mercado. Assim, o presente trabalho objetivou elaborar néctares de mamão com diferentes teores de açúcar, visando a adequar a doçura ideal em açúcar para esse produto. Foram elaboradas quatro formulações de néctar de mamão, com as mesmas proporções de polpa e água, e com concentrações de açúcar a 6, 8, 10 e 12\%. Cem julgadores não treinados participaram do teste afetivo de aceitação por meio da escala do ideal com nove categorias, variando de "extremamente menos doce que o ideal" a "extremamente mais doce que o ideal". Houve um aumento no percentual para a categoria "doçura ideal", conforme aumento da concentração de açúcar de 6 para 12\%. Foi verificada também uma predominância na frequência de respostas para as categorias "menos doce que o ideal”, para o néctar com 6\% de açúcar, e para as categorias "mais doce que o ideal", para o néctar com $12 \%$ de açúcar. Para a obtenção de um néctar de mamão com "doçura ideal”, a concentração de açúcar deve ser de 10,4\%.

Palavras-chave: aceitação sensorial; escala do ideal; doçura.

\section{ABSTRACT}

Papaya is a fruit whose pulp has attractive sensory, chemical and digestive characteristics, with high production in the country. Nevertheless, the nectar of this fruit is not widespread in the market. Thus, this study aimed to develop nectars of papaya with different sugar contents to adjust the sweetness to sugar ideal for this product. Four formulations of nectar of papaya were prepared with the same proportions of pulp and water, and sugar concentrations at 6, 8, 10 and $12 \%$. One hundred untrained panelists participated to the affective test of acceptance by the justabout-right scale with nine categories, ranging from "extremely less sweet than ideal" to "extremely sweeter than ideal". There was an increase in the percentage for the "ideal sweetness" as sugar concentration increased from 6 to 12\%. There was also verified a predominance in the frequency of responses for the categories "less sweet than ideal" to nectar of papaya with 6\% sugar and for the categories "sweeter than ideal" to nectar of papaya with $12 \%$ sugar. The sugar concentration must be $10.4 \%$ to obtain nectar papaya with "ideal sweetness".

Key words: sensory acceptability; just-about-right scale; sweetness.

\section{INTRODUÇÃO}

É crescente a busca dos consumidores por alimentos de qualidade, que sejam saudáveis e com pouca ou nenhuma adição de conservantes (BULL et al., 2004). Nesse aspecto, as frutas e produtos derivados, especialmente os in natura, têm se destacado. Pesquisas mostram que a fruticultura é responsável por mais de 505 milhões de toneladas produzidas mundialmente (FAO, 2012), sendo o Brasil o terceiro maior produtor (BRAZILIAN FRUIT, 2012). Aliado a isso, observa-se o crescimento da agroindústria frutícola, particularmente o setor de bebidas. De acordo com ROSA et al. (2006), o mercado interno brasileiro de sucos prontos para beber movimenta, em média, cerca de 250 milhões de litros por ano. Entretanto, segundo dados do Instituto Brasileiro de Frutas (IBRAF, 2011), em 2010, o consumo de sucos de todos os sabores perfez, aproximadamente, 550 milhões de litros somente no Brasil.

Dentre as distintas bebidas de frutas, a demanda pelos néctares tem crescido (ABIR, 2011).

\footnotetext{
'Instituto Federal de Educação, Ciência e Tecnologia do Triângulo Mineiro (IFTM), Ituiutaba, MG, Brasil.

IIDepartamento de Engenharia e Tecnologia de Alimentos, Instituto de Biociências, Letras e Ciências Exatas, Universidade Estadual Paulista “Júlio de Mesquita Filho”(UNESP), 15054-000, São José do Rio Preto, SP, Brasil. E-mail: contisil@ibilce.unesp.br. Autor para correspondência. Recebido 29.05.13 Aprovado 10.09.13 Devolvido pelo autor 22.01.14 CR-2013-0753.R1
} 
Para MATSUURA \& ROLIN (2002), tal aumento é consequente da falta de tempo da população, praticidade oferecida por esses produtos, substituição ao consumo de bebidas carbonatadas, valor nutritivo e preocupação com a ingestão de alimentos mais saudáveis. Segundo BRANCO et al. (2007), apesar do considerável aumento na ingestão desse produto, os sabores ainda mais consumidos são laranja, uva, maracujá, pêssego, manga e abacaxi. Entretanto, devido à diversidade de frutas no país e a miscigenação cultural, a procura por sabores diversificados tem levado ao desenvolvimento de novos produtos para atender à demanda.

O mamão é um fruto cuja polpa possui características sensoriais, químicas e digestivas atrativas (SHINAGAWA, 2009), destacando-se por seu teor em açúcares, pró-vitamina A, vitamina C (ARAÚJO FILHO et al., 2002), e por conter ferro, cálcio, magnésio e potássio (BLEINROTH \& SIGRIST, 1995). Segundo a FAO (2010), o mamão representa $10 \%$ da produção mundial de frutas, tendo se destacado entre os diferentes frutos tropicais (SANTANA et al., 2004). Apesar das altas produções contabilizadas nos últimos anos (IBGE, 2010), o que se observa é uma carência do néctar desse fruto no mercado. Nesse contexto, o presente trabalho objetivou elaborar distintas formulações de néctar de mamão, com diferentes teores de açúcar, visando a adequar a doçura ideal em açúcar por meio de teste afetivo de escala do ideal.

\section{MATERIAL E MÉTODOS}

Foram utilizadas polpas de mamão (Carica papaya L.) integral, previamente pasteurizadas e congeladas, fornecidas pela De Marchi Indústria e Comércio de Frutas Ltda. (Jundiaí, SP), água mineral potável sem gás Nativa (Goiânia, GO) e açúcar cristal de uso culinário.

O néctar de mamão, segundo Instrução Normativa 12/2003 do Ministério da Agricultura, Pecuária e Abastecimento (BRASIL, 2003), é uma bebida não fermentada, destinada ao consumo direto, obtida da dissolução da parte comestível do mamão ( $\boldsymbol{C}$. papaya L.) em água potável, adicionado de açúcares e opcionalmente ácidos, devendo conter, no mínimo, $35 \%$ de suco ou polpa e 6\% de açúcares totais. Por isso, foram elaboradas quatro formulações de néctar, com proporções de 6, 8, 10 e 12\% de açúcar, sendo fixado, em todas as formulações, o teor mínimo de polpa, conforme estabelecido em lei (Tabela 1). A porcentagem mínima de açúcar adicionada foi estabelecida em função da própria instrução normativa, e a porcentagem máxima foi determinada pelo fato de néctares comerciais, de diversos sabores, apresentarem, em seus rótulos, cerca de $12 \%$ de carboidratos (considerou-se que grande parte desses carboidratos seja proveniente de açúcar de adição).

Inicialmente, foi feito o descongelamento da polpa sob refrigeração a $7-10^{\circ} \mathrm{C}$, por 12 horas, e, posteriormente, foram adicionadas e misturadas as respectivas proporções de polpa, água e açúcar. Depois de elaborados, os produtos foram envasados em embalagens de vidro esterilizadas com capacidade de 500mL, tampados e armazenados sob refrigeração a $7-10^{\circ} \mathrm{C}$ até o momento da realização dos testes sensoriais. A elaboração e armazenamento dos néctares foram conduzidos na planta de processamento de vegetais e derivados do Instituto Federal de Educação, Ciência e Tecnologia do Triângulo Mineiro (IFTM), Câmpus Ituiutaba, seguindo estritamente as Boas Práticas de Fabricação.

Foi aplicado um teste sensorial afetivo de aceitação com cem julgadores não treinados, utilizando escala do ideal para doçura composta por nove categorias, variando de "extremamente menos doce que o ideal” a “extremamente mais doce que o ideal”. As amostras foram apresentadas de forma monádica, por meio de blocos completos e balanceados, em copos plásticos descartáveis de $50 \mathrm{~mL}$, codificados com três dígitos aleatórios. A quantidade servida foi padronizada em, aproximadamente, $30 \mathrm{~mL}$ por amostra e sob temperatura de $7-10^{\circ} \mathrm{C}$. O teste foi conduzido em cabines individuais, sob luz branca, no período da tarde, no Laboratório de Análise Sensorial do IFTM, Câmpus Ituiutaba.

Os resultados da avaliação sensorial foram analisados por meio de gráficos de distribuição de frequência das respostas. Para a determinação da concentração de açúcar, visando a obter a "doçura ideal” para o néctar de mamão, as nove categorias foram transformadas em escala numérica $(1=$ extremamente menos doce que o ideal, 5 = doçura ideal, 9 = extremamente mais doce que o ideal) e foi realizada análise de regressão linear simples entre a concentração de açúcar (variável independente) e os valores de ideal

Tabela 1 - Formulações dos néctares de mamão com 6, 8, 10 e $12 \%$ de açúcar.

\begin{tabular}{lllll}
\hline & & & & \\
Ingredientes & -----------------Formulações------------------- & & \\
& $6 \%$ & $8 \%$ & $10 \%$ & $12 \%$ \\
\hline polpa (g) & 1.050 & 1.050 & 1.050 & 1.050 \\
açúcar (g) & 180 & 240 & 300 & 360 \\
água (mL) & 1.770 & 1.710 & 1.650 & 1.590 \\
\hline
\end{tabular}


de doçura (variável resposta). Essas análises foram realizadas no software Excel, versão 2010.

\section{RESULTADOS E DISCUSSÃO}

Dos cem julgadores participantes, $48 \%$ pertenciam ao sexo feminino e $52 \%$ ao sexo masculino. A faixa etária variou dos 15 aos 48 anos, com média de 21 anos, predominando o público jovem (78\%), com idades entre 15 e 24 anos.

Observou-se um aumento no percentual para a categoria "doçura ideal”, conforme aumento da concentração de açúcar de 6 para 12\%, além de um deslocamento gradual no percentual de respostas das categorias "menos doce que o ideal”, para o néctar com $6 \%$ de açúcar, para as categorias "mais doce que o ideal”, para o néctar com 12\% de açúcar (Figura 1). Notou-se, também, que os néctares com $8 \%$ e, principalmente, com 10\% de açúcar apresentaram maiores frequências para as categorias próximas à “doçura ideal”. Apesar da formulação com 12\% de açúcar apresentar a maior frequência para a categoria “doçura ideal” (36\%), a alta porcentagem de notas para as categorias "mais doce que o ideal” já indica um excesso na quantidade de açúcar utilizado.
Apesar de este trabalho não ter avaliado a aceitação sensorial da doçura por meio de escala hedônica, mas por meio da escala de ideal de doçura, observa-se que os néctares de mamão com maior porcentagem de açúcar (10 e 12\%) apresentaram maiores indicações para a "doçura ideal” (Figura 1). Pesquisas indicam que os brasileiros têm uma maior preferência por produtos doces, com alto teor de açúcar (SOLOMON \& RIBEIRO, 2011). Em estudo realizado com cem julgadores não treinados, houve 81\% de aceitação para a doçura de bebidas de goiaba, adoçadas com $11 \%$ de sacarose (FERNANDES et al., 2009). Além disso, a faixa etária jovem (julgadores predominantes do atual estudo) e, em especial, a cultura mineira apresentam tal hábito alimentar (FISBERG, et al., 2000; ABDALA, 2006; MOMO et al., 2006; ESTIMA et al., 2009). Conforme MINIM (2010), as características não sensoriais devem ser levadas em consideração em estudos com consumidores, pois influem não somente na escolha de um produto, como também na aceitação e no processo de compra deste.

A análise de regressão linear entre a concentração de açúcar e os valores de ideal de doçura

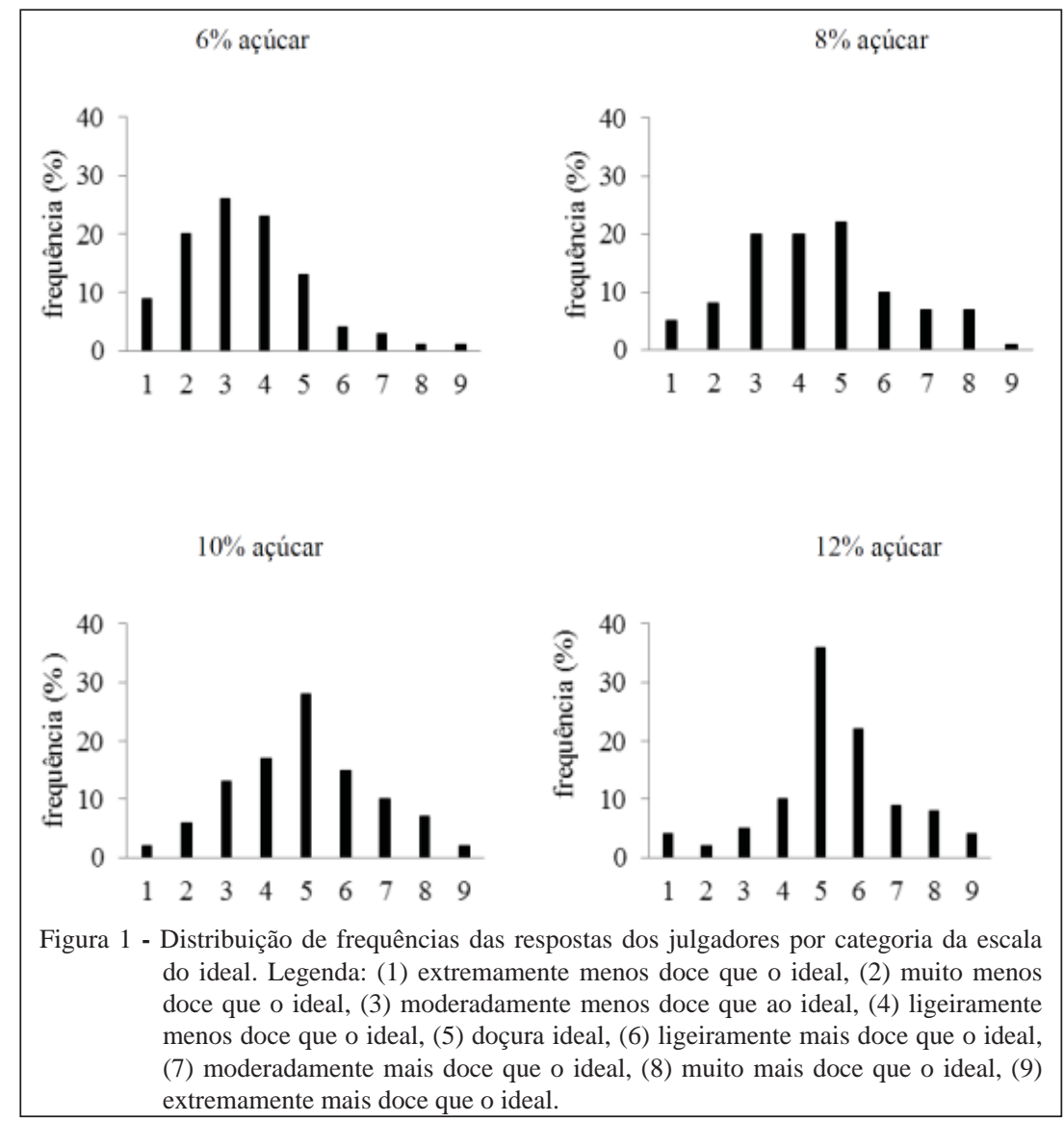

Ciência Rural, v.44, n.4, abr, 2014. 
indicaram “doçura ideal” para o néctar de mamão contendo 10,4\% de açúcar, ponto este em que a reta apresentada na figura 2 cruza o eixo Y no valor 5, referente à doçura ideal. Apesar da maior frequência de "doçura ideal” para o néctar com $12 \%$ de açúcar (Figura 1), a distribuição de frequências ao redor da categoria "doçura ideal” para o néctar com 10\% de açúcar é mais homogênea e há altas porcentagens para as categorias “mais doce que o ideal” para o néctar com $12 \%$, como já explicado anteriormente, sendo estes os fatores responsáveis pela concentração de açúcar considerada como ideal estar próxima ao valor de $10 \%$, e não de $12 \%$.

Outros trabalhos apontam resultados semelhantes para o ideal de açúcar em sucos e néctares. BRITO et al. (2007) encontraram um teor ideal em sacarose de 9,6\% em diferentes amostras de néctar de goiaba, apresentadas a trinta julgadores não treinados, consumidores do produto. MARCELLINI et al. (2005) avaliaram o ideal de doçura em suco de abacaxi industrializado, reconstituído e adoçado com sacarose, em concentrações de 5 a 17,5\%, e verificaram que o suco com $8,39 \%$ foi o considerado mais ideal. FREITAS \& MATTIETTO (2013) encontraram 9,5\% e 10,7\%, respectivamente, para concentração ótima de açúcar em blends de cupuaçuacerola-açaí e graviola-camucamu-taperabé (frutos típicos da Amazônia), ao empregar escala do ideal com consumidores locais.

\section{CONCLUSÃO}

Há uma predominância na frequência de respostas para as categorias "menos doce que o ideal”, para o néctar de mamão com 6\% de açúcar, e para as categorias “mais doce que o ideal”, para o néctar com 12\% de açúcar, havendo um aumento na frequência

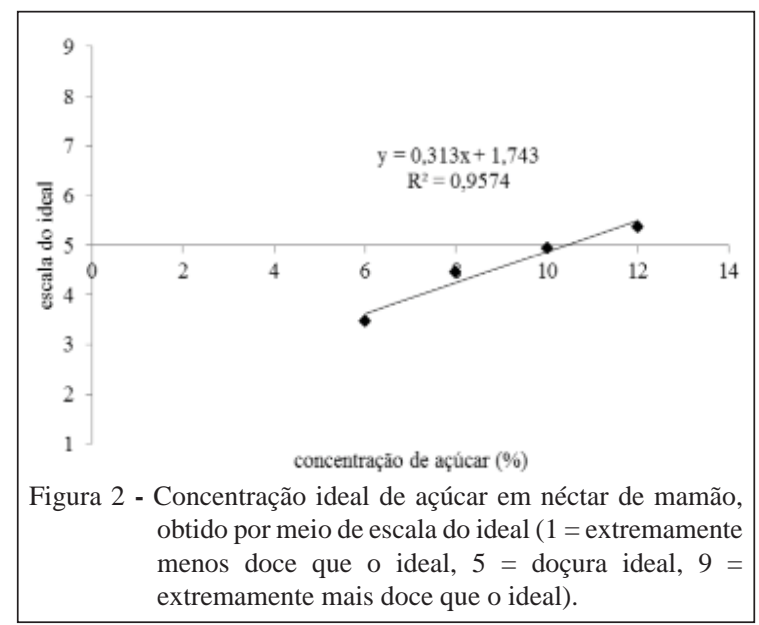

de respostas para a categoria “doçura ideal”, quando do aumento na concentração de 6 a 12\% de açúcar. Para a obtenção de um néctar de mamão com "doçura ideal”, a concentração de açúcar deve ser de 10,4\%.

\section{AGRADECIMENTOS}

Os autores agradecem à De Marchi Indústria e Comércio de Frutas Ltda., pelo fornecimento da polpa congelada de mamão.

\section{COMITÊ DE ÉTICA E BIOSSEGURANÇA}

O projeto foi aprovado pelo Comitê de Ética em Pesquisa do Instituto de Biociências, Letras e Ciências Exatas da Universidade Estadual Paulista "Júlio de Mesquita Filho", de acordo com a Resolução CNS/196/96, por meio do parecer de número 123.364.

\section{REFERÊNCIAS}

ABDALA, M.C. Sabores da tradição. Revista do Arquivo Público Mineiro, v.1, p.118-129. 2006. Disponível em: <http:// www.siaapm.cultura.mg.gov.br/acervo/rapm_pdf/ Sabores_da_ tradicao.PDF>. Acesso em: 24 fev. 2013.

ARAÚJO FILHO, G.C. et al. Produtor de mamão. Fortaleza: Edições Demócrito Rocha, Instituto Centro de Ensino Tecnológico, 2002. 72p.

ASSOCIAÇÃO BRASILEIRA DAS INDÚSTRIAS DE REFRIGERANTES E BEBIDAS NÃO ALCOÓLICAS (ABIR). 2011. Disponível em: <http://abir.org.br/categoria/o-setor/>. Acesso em: 19 jul. 2012.

BLEINROTH, E.W.; SIGRIST, J.M.M. Matéria-prima. In: MEDINA, J.C. Mamão: cultura, matéria- prima, processamento e aspectos econômicos. Campinas: ITAL, Frutas Tropicais, 1995. Cap.7, p.179-254.

BRANCO, I.G. et al. Avaliação sensorial e estabilidade físicoquímica de um blend de laranja e cenoura. Ciência e Tecnologia de Alimentos, v.27, n.1, p.7-12, 2007. Disponível em: <http:// www.scielo.br/pdf/cta/v27n1/01.pdf>. Acesso em: 24 maio 2013. doi: 10.1590/S0101-20612007000100002.

BRASIL. Ministério da Agricultura, Pecuária e Abastecimento. Instrução Normativa n. 12, de 04 de setembro de 2003. Regulamento técnico para os padrões de identidade e qualidade dos néctares de abacaxi, acerola, cajá, caju, goiaba, graviola, mamão, manga, maracujá, pêssego e pitanga. Anexo III. Diário Oficial da União, Brasília, DF, 2003. Disponível em: <http:// extranet.agricultura.gov.br/sislegis-consulta/consultarLegislacao. do ?operacao= visualizar\&id=2831 $>$. Acesso em: 19 jul. 2012.

BRITO, C.A.K. et al. Equivalência de dulçor e poder edulcorante de néctares de goiaba adoçados com diferentes edulcorantes. Revista Brasileira de Tecnologia Agroindustrial, v.1, n.2, p.2636, 2007. Disponível em: <http://revistas.utfpr.edu.br/pg/index. php/rbta/article/view/257/225>. Acesso em: 24 maio 2013.

BULL, M.K. et al. The effect of high pressure processing on the microbial, physical and chemical properties of Valencia and 
Navel orange juice. Innovative Food Science and Emerging Technologies, v.5, n.2, p.135-149, 2004. Disponível em: <http:// dx.doi.org/10.1016/j.ifset.2003.11.005>. Acesso em: 24 jan. 2013. doi: 10.1016/j.ifset.2003.11.005.

ESTIMA, C.C.P. et al. Meal consumption patterns and anthropometric measurements in adolescents from a low socioeconomic neighborhood in the metropolitan area of Rio de Janeiro, Brazil. Appetite, v.52, p.735-739, 2009. Disponível em: $<$ http://www.ncbi.nlm.nih.gov/pubmed/19501773>. Acesso em: 24 maio 2013. doi: 10.1016/j.appet.2009.03.017.

FERNANDES, A.G. et al. Avaliação sensorial de bebidas de goiaba adoçadas com diferentes agentes adoçantes. Ciência e Tecnologia de Alimentos, v.29, n.2, p.358-364, 2009. Disponível em: <http://www.scielo.br/pdf/cta/v29n2/19.pdf>. Acesso em: 24 maio 2013. doi: 10.1590/S0101-20612009000200019.

FISBERG, M. et al. Hábitos alimentares na adolescência. Pediatria Moderna, v.36, n.11, p.724-734, 2000. Disponível em: <http://www.moreirajr.com.br/revistas.asp ?fase=r003\&id_ materia=203>. Acesso em: 24 jan. 2013.

FOOD AND AGRICULTURE ORGANIZATION OF UNITED NATIONS (FAO/FAOSTAT). 2010. Disponível em: <http:// faostat.fao.org/>. Acesso em: 24 maio 2013.

FOOD AND AGRICULTURE ORGANIZATION OF UNITED NATIONS (FAO/FAOSTAT). 2012. Disponível em: <http:// faostat.fao.org/>. Acesso em: 19 jul. 2012.

FREITAS, D. De G.C.; MATTIETTO, R.A. Ideal sweetness of mixed juices Amazon fruits. Ciência e Tecnologia de Alimentos, v.33, supl.1, p.148-154, 2013. Disponível em: <http://www.scielo. br/scielo.php?pid=S0101-20612013000500022\&script $=$ sci arttext \&tlng=en>. Acesso em: 24 maio 2013. doi: 10.1590/ S0101-20612013000500022.

INSTITUTO BRASILEIRO DE FRUTAS (IBRAF). 2011. Disponível em: <http://www.ibraf.org.br/>. Acesso em: 31 ago. 2011.

INSTITUTO BRASILEIRO DE GEOGRAFIA E ESTATÍSTICA (IBGE). 2010. Disponível em: <http://www.ibge.gov.br/>. Acesso em: 24 maio 2013.

MARCELLINI, P.S. et al. Doçura ideal e análise de aceitação de suco de abacaxi concentrado reconstituído adoçado com diferentes edulcorantes e sacarose. Alimentos e Nutrição, v.16, n.2, p.177-182, 2005. Disponível em: <http://serv-bib.fcfar.unesp.br/seer/index.php/ alimentos/article/viewFile/318/308>. Acesso em: 12 jan. 2013.
MATSUURA, F.C.A.U.; ROLIM, R. Avaliação da adição de suco de acerola em suco de abacaxi, visando a produção de um "blend" com alto teor de vitamina C. Revista Brasileira de Fruticultura, v.24, n.1, p.138-141, 2002. Disponível em: $<$ http://www.scielo.br/scielo.php?script=sci_arttext\&pid $=$ S0100-29452002000100030 $>$. Acesso em: 24 maio 2013. doi: 10.1590/S0100-29452002000100030.

MINIM, V.P.R. Análise sensorial: estudos com consumidores. 2.ed. rev. e ampl. Viçosa: UFV, 2010. 308p.

MOMO, C.A. et al. Avaliação da dieta habitual de escolares de Piracicaba: aplicação do questionário de frequência alimentar para adolescentes (QFAA) reestruturado em estudo-piloto. Segurança Alimentar e Nutricional, v.13, n.1, p.38-48, 2006. Disponível em:<http://www.unicamp.br/nepa/arquivo_san/avaliacao_de_ dieta.pdf>. Acess o em: 20 fev. 2013.

PROGRAMA DE PROMOÇÃO DAS EXPORTAÇÕES DAS FRUTAS BRASILEIRAS E DERIVADOS (BRAZILIAN FRUIT). 2012. Disponível em: <http://www.brazilianfruit.org/ Pbr/Fruticultura/ Fruticultura.asp>. Acesso em: 19 jul. 2012.

ROSA, S.E.S. et al. Panorama do setor de bebidas no Brasil. BNDES Setorial, n.23, p.101-150, mar. 2006. Disponível em: <http://www.bndes.gov.br/SiteBNDES/ export/sites/default/ bndes_pt/Galerias/Arquivos/conhecimento/bnset/set2304.pdf>. Acesso em: 24 maio 2013.

SANTANA, L.R.R. et al. Genótipos melhorados de mamão (Carica papaya L.): avaliação sensorial e físico-química dos frutos. Ciência e Tecnologia de Alimentos, v.24, n.2, p.217222, 2004. Disponível em: <http://www.scielo.br/pdf/cta/v24n2/ v24n2a10.pdf>. Acesso em: 01 jan. 2013. doi: 10.1590/S010120612004000200010.

SHINAGAWA, F.B. Avaliação das características bioquímicas da polpa de mamão (Carica papaya $\mathrm{L}$.) processada por alta pressão hidrostática. 2009. 133f. Dissertação (Mestrado em Tecnologia de Processos Químicos e Bioquímicos) - Curso de Pósgraduação em Tecnologia de Processos Químicos e Bioquímicos, Escola de Química, Centro de Tecnologia, Universidade Federal do Rio de Janeiro, RJ.

SOLOMON, M.R.; RIBEIRO, L.B. O comportamento do consumidor: comprando, possuindo e sendo. 9.ed. Porto Alegre: Bookman, 2011. 446p. 